\title{
On the Behaviour in Scale-Space of Local Extrema and Blobs
}

\author{
Tony Lindeberg \\ Computational Vision and Active Perception Laboratory (CVAP) \\ Department of Numerical Analysis and Computing Science \\ Royal Institute of Technology \\ S-100 44 Stockholm, Sweden \\ Email: tony@bion.kth.se
}

\begin{abstract}
We apply elementary techniques from real analysis and singularity theory to derive analytical results for the behaviour in scale-space of critical points and related entities. The main results of the treatment comprise:

- a description of the general nature of trajectories of critical points in scale-space.

- an estimation of the drift velocity of critical points and edges.

- an analysis of the qualitative behaviour of critical points in bifurcation situations.

- a classification of what types of blob bifurcations are possible.
\end{abstract}




\section{Introduction}

It is well-known that scale-space smoothing leads to shape distortions. For example, features like local extrema, edges, blobs etc can be expected to drift when the underlying grey-level image is subject to blurring.

Aspects of this phenomenon have been studied by other authors from different viewpoints. Canny [6] discussed the general trade-off problem between detection and localization occurring in edge detection. Bergholm [4] estimated the drift velocity of edges for a set of plausible configurations with the aim of estimating a scale step for the edge focusing algorithm. Berzins [5] has analysed the localization error for zero-crossings of the Laplacian.

Other kinds of phenomena affecting the topology can also occur. Blobs can disappear, merge and split as developed by Koenderink and van Doorn [8]. Similar kinds of transitions apply to edges, zero-crossings of the Laplacian, corners etc.

In this work we will perform a study of critical points, that is local extrema and saddle points, and analyse in detail what happens to those features when the underlying image undergoes scalespace smoothing. We will essentially (1) develop how these feature points can be expected to behave when the scale parameter in scale-space changes, (2) derive an expression for their drift velocity and (3) classify their behaviour at bifurcations into a discrete set of generic cases, which will also result in a classification of the behaviour of grey-level blobs and scale-space blobs at bifurcation points. The results to be presented are not based on any specific models of the intensity variations in the image but are expressed on a general form under rather weak a priori assumptions.

The scale-space concept we will deal with is the traditional diffusion based scale-space for continuous signals developed by Witkin [16], Koenderink, van Doorn [7, 8] and Babaud et al. [3], which is given by the solution to the diffusion equation

$$
\frac{\partial L}{\partial t}=\frac{1}{2}\left(\frac{\partial^{2} L}{\partial x^{2}}+\frac{\partial^{2} L}{\partial y^{2}}\right)
$$

with initial condition $L(\cdot ; 0)=f(\cdot)$ where $f$ indicates the original signal.

\section{Trajectories of Critical Points in Scale-Space}

In many situations it is of interest to estimate the drift velocity of critical points when the scale parameter varies. Such information is useful for instance when estimating the localization error of feature points due to blurring or when tracking local extrema or related entities between scales as done for instance by Lifshitz and Pizer [9] or in the scale-space primal sketch developed by Lindeberg and Eklundh $[11,12,13]$. In non-degenerate situations, that is when the second differential is a non-degenerate quadratic form, we can base such an analysis on the implicit function theorem.

Definition 1 (Critical point (2D))

A point $\left(x_{0}, y_{0}\right)$ is a critical point of a mapping $f: R^{2} \rightarrow R$ if the gradient in this point is zero. The critical point is said to be non-degenerate if the Hessian matrix in this point is non-singular. Otherwise it is called degenerate.

Lemma 1 (Behaviour of critical points in continuous scale-space (2D))

Let $L: R^{2} \times R \rightarrow R$ be the scale-space representation of a two-dimensional continuous signal given by the diffusion equation (1). Assume that at some scale level $t_{0}>0$ the point $\left(x_{0}, y_{0}\right)$ is a non-degenerate critical point for the mapping $(x, y) \mapsto L\left(x, y ; t_{0}\right)$.

Then there exist an open set $S_{\left(x_{0}, y_{0} ; t_{0}\right)} \subset R^{2} \times R$ and an open interval $I_{t_{0}} \subset R$ with $\left(x_{0}, y_{0} ; t_{0}\right) \in S_{\left(x_{0}, y_{0} ; t_{0}\right)}$ and $t_{0} \in I_{t_{0}}$ having the following property: To every $t_{1} \in I_{t_{0}}$ there corresponds a unique $\left(x_{1}, y_{1}\right)$ such that $\left(x_{1}, y_{1} ; t_{1}\right) \in S_{\left(x_{0}, y_{0} ; t_{0}\right)}$ and $\left(x_{1}, y_{1}\right)$ is a non-degenerate critical point for the mapping $(x, y) \mapsto L\left(x, y ; t_{1}\right)$. 
If this $\left(x_{1}, y_{1}\right)$ is defined to be $r\left(t_{1}\right)$ then $r$ is a continuously" differentiable mapping $I_{t_{0}} \rightarrow R^{2}$ such that

- $r\left(t_{0}\right)=\left(x_{0}, y_{0}\right)$

- $r\left(t_{1}\right)$ is for every $t_{1} \in I_{t_{0}}$ a non-degenerate critical point for the mapping $(x, y) \mapsto L\left(x, y ; t_{1}\right)$.

- the derivative of $r$ with respect to $t$ in the point $\left(x_{0}, y_{0}\right)$ is given by

$$
\frac{d r}{d t}\left(t_{0}\right)=-\frac{1}{2}\left(\begin{array}{cc}
\frac{\partial^{2} L}{\partial x^{2}} & \frac{\partial^{2} L}{\partial y \partial x} \\
\frac{\partial^{2} L}{\partial x \partial y} & \frac{\partial^{2} L}{\partial y^{2}}
\end{array}\right)_{\left(x_{0}, y_{0}\right)}^{-1}\left[\left(\frac{\partial^{2}}{\partial x^{2}}+\frac{\partial^{2}}{\partial y^{2}}\right)\left(\begin{array}{c}
\frac{\partial L}{\partial L} \\
\frac{\partial L}{\partial y}
\end{array}\right)\right]_{\left(x_{0}, y_{0}\right)}
$$

Proof: Follows easily by applying the implicit function theorem to the gradient function. See [14] for the details. $\diamond$

A corresponding result does of course also hold in one dimension. (Just remove all occurrences of $y$ ). In that case the derivative of $r: I_{t_{0}} \rightarrow R$ reduces to

$$
\frac{d r}{d t}\left(t_{0}\right)=-\frac{1}{2} \frac{\frac{\partial^{3} L}{\partial x^{3}}\left(x_{0} ; t_{0}\right)}{\frac{\partial^{2} L}{\partial x^{2}}\left(x_{0} ; t_{0}\right)}
$$

\section{$2.1 \quad$ Interpretations}

This lemma expresses how critical points in general can be expected to behave in scale-space. The most immediate interpretation is that it gives a straightforward estimation of the drift velocity of critical points in under scale-space smoothing.

Proposition 2 (Drift velocity of critical points in scale-space (2D))

Given the scale-space representation $L: R^{2} \times R_{+} \rightarrow R$, of a two-dimensional signal assume that for some scale level $t_{0}>0$ the point $\left(x_{0}, y_{0}\right)$ is a non-degenerate critical point for the mapping $(x, y) \mapsto L\left(x, y ; t_{0}\right)$. Then the drift velocity of that critical point when the scale parameter changes is given by (2).

Note that the drift velocity can actually become infinite when the the Hessian becomes singular. At such points bifurcations can occur, as will be developed in Section 3.

\section{Corollary 3 (Unbounded drift velocity of critical points)}

The drift velocity of critical points tends to infinity near bifurcations.

These conclusions are of course valid also in one dimension. The expression for the drift velocity is, however, much simpler in this case:

Proposition 4 (Drift velocity of critical points in scale-space (1D))

Given the scale-space representation $L: R \times R_{+} \rightarrow R$, assume that for some scale level $t_{0}>0$ the point $x_{0}$ is a non-degenerate critical point for the mepping $x \mapsto L\left(x ; t_{0}\right)$. Then the drift velocity of that critical point as the scale parameter changes is given by (3).

This estimation can easily be extended to comprise edges as well. For simplicity, assume that the edge under study is sufficiently long and sufficiently close to a straight line such that a one-dimensional analysis is a valid approximation. Further, without loss of generality assume that the coordinate system is oriented such that the edge is perpendicular to the $x$-axis. Then, we can use for instance non-maximum suppression to define the location of the edge as those points where the first derivative in the gradient direction (that is here the $x$-direction) has a local maximum. In other words, the edge is defined by those points where the second derivative along the gradient direction is zero. Now, since under these conditions, critical points are given by zeros in the first derivative and edge points by zeros in the second derivative, we can apply Proposition 4 to this situation just by replacing $L$ by $\frac{\partial L}{\partial x}$. Hence, 
Proposition 5 (On the drift velocity of straight edges in scale-space (2D)) Given the scale-space representation $L: R^{2} \times R_{+} \rightarrow R$, assume that for some scale level $t_{0}>0$ the point $\left(x_{0}, y_{0}\right)$ is an edge point along a long straight line. Moreover, assume that the coordinate system is aligned to the edge such that the $x$-direction is perpendicular to the edge and further that the third derivative in this direction is non-zero. Then the drift velocity of the edge point as the scale parameter changes is given by

$$
\frac{d r}{d t}\left(t_{0}\right)=-\frac{1}{2} \frac{\frac{\partial^{4} L}{\partial x^{4}}\left(x_{0}, y_{0} ; t_{0}\right)}{\frac{\partial x^{3} L}{\partial x^{3}}\left(x_{0}, y_{0} ; t_{0}\right)}
$$

This result is applicable also to edges given by zero-crossings provided that the second derivative along the direction of the edge (here the $y$-direction) is sufficiently small to be neglected. Trivially, an identical result holds for the edges of a one-dimensional signals. Note, that we have not made any specific assumptions about the shape of the intensity profile perpendicular to the edge.

\section{Corollary 6 (Unbounded drift velocity of straight edges)}

The drift velocity of edges tends to infinity when two adjacent parallel edges are just about to merge into one.

These result can, for instance, be used for explaining a recent observation by Zhang and Bergholm [17], where they noted that configurations consisting of two adjacent edges (so-called "staircase edges", see Figure 1) can lead to a rapid edge drift, which in turn violates the assumptions behind the step size estimates used in the edge focusing algorithm [4]. In this situation the third derivative is in fact very close to zero, and a bifurcation just about to take place.
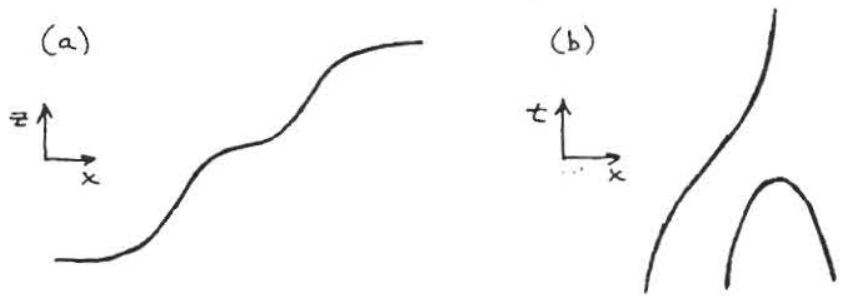

Figure 1: (a) A configuration consisting of a double step edge can lead to a rapid edge drift. (b) This behaviour can be explained by considering the zero-crossings paths under variations in scale. When the edge points tend to each other the drift velocity tends to infinity. See also Section 3 for a more detailed description of what will happen at bifurcations.

Another consequence of Lemma 1 and its one-dimensional analogy is that a non-degenerate critical point existing at a certain level of scale can in general be traced to a similar critical point both at a slightly coarser and a slightly finer scale. By continuation, such local paths obtained from the implicit function theorem, can be extended to curves as long as the Hessian determinant remains non-zero. Hence, we get trajectories of critical points that in general will be regular curves, delimited from above and below by some scale values, $t_{\min }$ and $t_{\max }$, at which the Hessian is zero and the critical point hence degenerate. One easily shows, see [14], that the type of critical point will not change along such a path. Therefore, we define:

Proposition 7 (Extremal paths and saddle paths (2D))

Given the scale-space representation $L: R^{2} \times R_{+} \rightarrow R$, assume that for some scale level $t_{0}>0$ the point $\left(x_{0}, y_{0}\right)$ is a non-degenerate maximum (minimum/saddle) point for the mapping $(x, y) \mapsto L\left(x, y ; t_{0}\right)$. Then there exists a unique trajectory of maximum (minimum/saddle) points $r: I_{t_{0}} \rightarrow R^{2}$ with $t_{0} \in I_{t_{0}}$ such that $r\left(t_{0}\right)=\left(x_{0}, y_{0}\right)$ and $r(t)$ is for every $t$ a local maximum (minimum/saddle) point for the mapping $(x, y) \mapsto L(x, y ; t)$. This trajectory is called the extremal (extremal/saddle) path through $\left(x_{0}, y_{0} ; t_{0}\right)$ and is denoted by $M^{+}\left(x_{0}, y_{0} ; t_{0}\right)$ $\left(M^{-}\left(x_{0}, y_{0} ; t_{0}\right) / S\left(x_{0}, y_{0} ; t_{0}\right)\right)$. The associated scale interval, where the path is defined, is delimited by a minimum scale $t_{\min }$ and a maximum scale $t_{\max }$. At those scalcs the critical paths end up in degenerate critical points unless the minimum scale is zero or the maximum scale infinite. At all interior points the associated critical points are non-degenerate. 
The one-dimensional situation is similar, although simpler, since there are no stable saddle points in this case.

\section{Behaviour Near Singularities: Classification}

The results so far describe the evolution properties in scale-space of non-degenerate critical points. When we are to investigate the behaviour of degenerate critical points, the approach with the implicit function theorem is no longer applicable, since at those points the Hessian matrix will be singular.

Useful methods for analysing such points, which generically will be isolated, can be obtained from a branch of mathematics known as singularity or catastrophe theory, see e.g. Poston and Stewart [15] for an application-oriented introduction and e.g Arnold et al. [1, 2] for a more rigorous treatment. One of the fundamental results in this field is that the typical qualitative behaviour of families with a small number of parameters can be expressed completely by the qualitative behaviour of a finite set of families. A famous theorem by Thom classifies the behaviour of families of functions with the number of parameters $r \leq 4$ into seven elementary catastrophes, see e.g. Poston and Stewart [15]. For one-parameter families of functions the result can be summarized as:

Thom's classification theorem applied to one-parameter families:

Typically a one-parameter family $R^{n} \times R \rightarrow R$ of smooth functions $R^{n} \rightarrow R$, for any $n$, is structurally stable and is in every point (locally) equivalent to one of the following forms ${ }^{1}$ :

- non-critical: $x_{1}$

- non-degenerate critical, or Morse: $x_{1}^{2}+\ldots+x_{i}^{2}-x_{i+1}^{2}-\ldots-x_{n}^{2}(0 \leq i \leq n)$

- degenerate critical - fold catastrophe $\left(A_{2}\right): x_{1}^{3}+u_{1} x_{1} \pm x_{2}^{2} \pm \ldots \pm x_{n}^{2}$

The $A_{2}$ type of singularity in the fold singularity means that the first and second derivatives in one direction are zero while the third derivative in that direction is non-zero. At the singularity point the function is locally equivalent to the function $x^{3} \pm y^{2}$ and from the concept of equivalence between families of functions it follows that any transversal unfolding through a singularity of this type will be equivalent to the family $G_{1}(x, y ; u)=x^{3}+u x \pm y^{2}$, where $x$ and $y$ should be interpreted as state variables and $u$ serve as the parameter.

Therefore, if one is interested in the behaviour of the critical points of a signal during the evolution of the diffusion equation, it should in principle be sufficient to study this situation. For two-dimensional signals the singularity set is given by the solutions of

$$
\begin{aligned}
& \frac{\partial G_{1}}{\partial x}(x, y ; u)=3 x^{2}+u=0 \\
& \frac{\partial G_{1}}{\partial y}(x, y ; u)= \pm 2 y=0
\end{aligned}
$$

We easily observe that the singularity set is given by $\left(x_{1}(u), y_{1}(u)\right)=\left(-\sqrt{-\frac{u}{3}}, 0\right)$ and $\left(x_{2}(u), y_{2}(u)\right)=$ $\left(+\sqrt{-\frac{u}{3}}, 0\right)(u \leq 0)$. From the sign of the Hessian determinant $\left|\left(\mathcal{H} G_{1}\right)\right|(x, y ; u)= \pm 12 x$ it follows that $\left(x_{1}(u), y_{1}(u)\right)$ are saddle/maximum points and $\left(x_{2}(\imath \iota), y_{2}(u)\right)$ are minimum/saddle points for every $u<0$. At the (isolated) bifurcation point corresponding to $u=0$ the critical points merge and then disappear, see also Figure 2.

\footnotetext{
${ }^{1}$ Equivalence between two families of functions essentially means that one of the families can be smoothly transformed into the other family by a set of diffeomorphisms. This means that singularity sets, which are the sets of points where the first differential with respect to the state variables $\left(\in R^{n}\right)$ are zero, will also be qualitatively similar. Intuitively, the notion of structural stability, actually transversality, means that the qualitative behaviour of the family will not be affected by (sufficiently) small perturbations. See e.g. Poston and Stewart (1978) or Lindeberg (1991) for a more precise treatment.
} 
By similar arguments the scale-space representation of a one-dimensional signal will at a bifurcation point be locally equivalent to the unfolding $G_{1}(x ; u)=x^{3}+u x$. The same type of calculations as above show that in this case the fold catastrophe instead describes the merging of a maximum point and a minimum with increasing $u$, see also Figure 2. To summarize,

Corollary 8 (Generic behaviour at singularities in continuous scale-space (2D)) The typical behaviour to be expected at the singularities in a one-parameter family of twodimensional signals are annihilations or creations of pairs of local extrema and saddle points.

Corollary 9 (Generic behaviour at singularities in continuous scale-space (1D)) The typical behaviour to be expected at the singularities in a one-parameter family of onedimensional signals are annihilations or creations of pairs of local maxima and local minima.

Observe in this context that in the scale-space representation of a one-dimensional signal the number of local extrema cannot increase when the scale parameter increases, see e.g. [10]. This means that creations of pairs of local maxima and minima with increasing scale are impossible if the special structure of the diffusion equation is taken into account. However, as will be demonstrated below, creations of saddle-extremum pairs with increasing scale are possible in the scale-space representation of a two-dimensional signal.
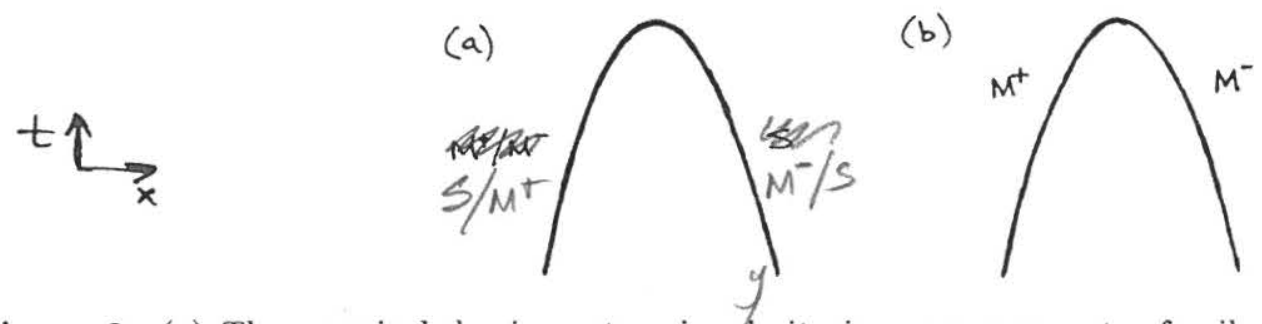

Figure 2: (a) The generic behaviour at a singularity in a one-parameter family of two-dimensional functions can be described by the unfolding $G_{1}(x ; u)=x^{3}+u x \pm y^{2}$. The singularity set of this family, that is the set of critical points to the mappings $(x, y) \mapsto G_{1}(x, y ; u)$, describes an extremum point and a saddle point merging along a parabola and then disappearing. (b) For a one-parameter family of one-dimensional functions the behaviour is instead given by $G_{1}\left(x_{j} u\right)=x^{3}+u x$. The singularity set in this case corresponds to a similar merge of a maximum point and a minimum point.

\subsection{Classification of Blob Events at Bifurcations in Scale-Space}

A natural question that arises in connection with the scale-space primal sketch concerns which types of blob events are possible in bifurcation situations. Since scale-space blobs are defined as families of grey-level blobs (see [11,12] and Figure 5) and grey-level blobs in turn are defined by pairs of critical points (see [11,12] and Figure 3) it follows that the behaviour of a scale-space blob near a singularity will be solely determined by the behaviour of its associated critical paths during a short scale interval around the bifurcation moment. Compared to the previous analysis, where we were dealing with critical points only, we must however take into account the fact that a saddle point delimiting the extent of a grey-level blob involved a bifurcation, can be associated with other grey-level blobs as well. This leads to natural coupling between scale-space blobs sharing the same saddle path in a neighbourhood of a bifurcation. We define:

Definition 2 (Non-shared saddle path (2D))

Given a saddle path involved in a structurally stable bifurcation of a two-dimensional signal we say that the saddle path is non-shared before (after) the bifurcation if there exists some scale interval before (after) the bifurcation during which every saddle point of the saddle path is not contained in more than one grey-level blob. Otherwise, the saddle path is said to be shared.

More formally, a saddle path is said to be non-shared before (after) a bifurcation at $t_{\text {bifurc }}$ if there exists some $\epsilon>0$ such that for all scales in the interval $t \in] t_{\text {bifurc }}-\epsilon, t_{\text {bifurc }}\left[(t \in] t_{\text {bifurc }}, t_{\text {bifurc }}+\epsilon[)\right.$ 

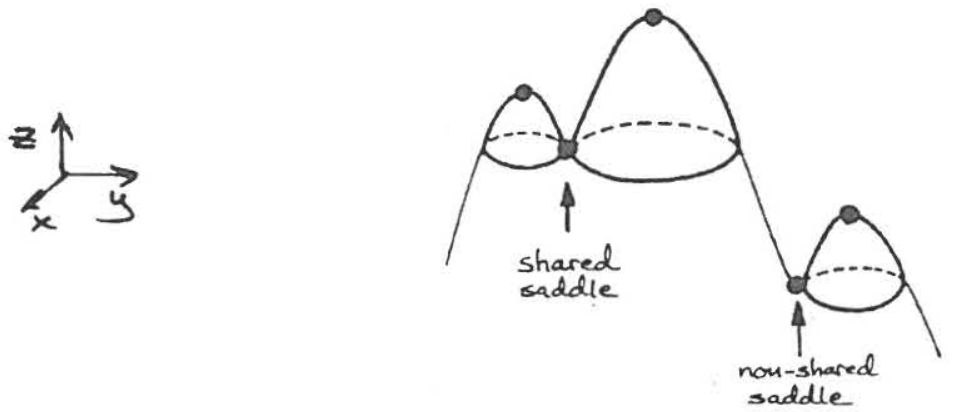

Figure 3: Illustration of the definition of grey-level blob for a two-dimensional signal. Every local extremum gives rise to a blob and the extent of the blob is given by a saddle point. A saddle point is said to be shared if it is contained in more than one grey-level blob.

the saddle point of the saddle path at that scale does not belong to more than one grey-level blob, see also Figure 3.

This definition implies that a non-shared saddle path, participating in say an extremumsaddle pair disappearing with increasing scale, describes an isolated blob that disappears. We call this event a blob annihilation. On the other hand, a shared saddle path involved in a similar event describes a blob disappearing under the influence of a neighbour blob, a blob merge. Taking the classification in Corollary 8 into account, we therefore have: (Below, the term annihilation (creation) of an extremum-saddle pair means that a pair consisting of an extremum path and a saddle path disappears (appears) when the scale parameter increases.)

Proposition 10 (Scale-space blob events (2D))

In the scale-space representation of a two-dimensional continuous signal, the following blob events are possible at a structurally stable bifurcation:

- blob annihilation - annihilation of an extremum-saddle pair where the saddle path is nonshared before the bifurcation.

- blob merge - annihilation of an extremum-saddle pair where the saddle path is shared with another scale-space blob before the bifurcation.

- blob split - creation of an extremum-saddle pair where the saddle path is shared with another scale-space blob after the bifurcation.

- blob creation - creation of an extremum-saddle pair where the saddle path is non-shared after the bifurcation.

These four types constitute the definitions of the terms aninihilation, merge, split and creation with respect to grey-level blobs and scale-space blobs in the two-dimensional case.

Proof: From Corollary 8 we have that the typical behaviour at singularities are pairwise annihilations and creations of extremum-saddle pairs. Combined with the definition of shared saddle path this means that for structurally stable bifurcations, the class of possible blob events is restricted to the given four types.

What remains to verify is that all these four types can be instantiated and that they also are structurally stable. It is well-known that blob annihilations and blob merges can take place in scale-space. Mlustrative examples, also indicating the more detailed quantitative behaviour around singularities, are given in [14]. The fact that splits can occur is also known, see e.g. the example given by Lifshitz and Pizer [9] illustrated in Figure 4 and [10]. The latter configuration can also be modified to describe a blob creation as well, if the higher one of the two peaks is replaced by a double peak, see Figure 4 . Then the extent of the two smaller blobs at the higher peak will be delimited by the grey-level in the valley between them, which means that when the narrow ridge has eroded enough to give rise to the creation of a saddle-extremum pair, then the saddle path in the created saddle-extremum pair will not be shared by any other blob. $\diamond$

The assumption of structural stability is important in this context, since otherwise, there is an infinite variety of possible events. For instance, three or more blobs could merge into one blob 

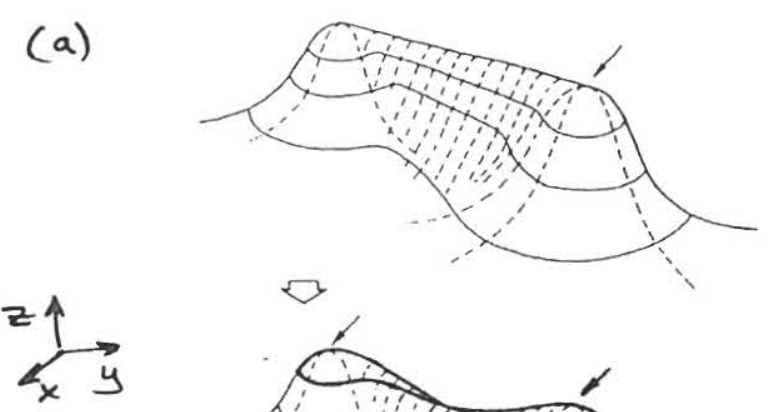

(b)

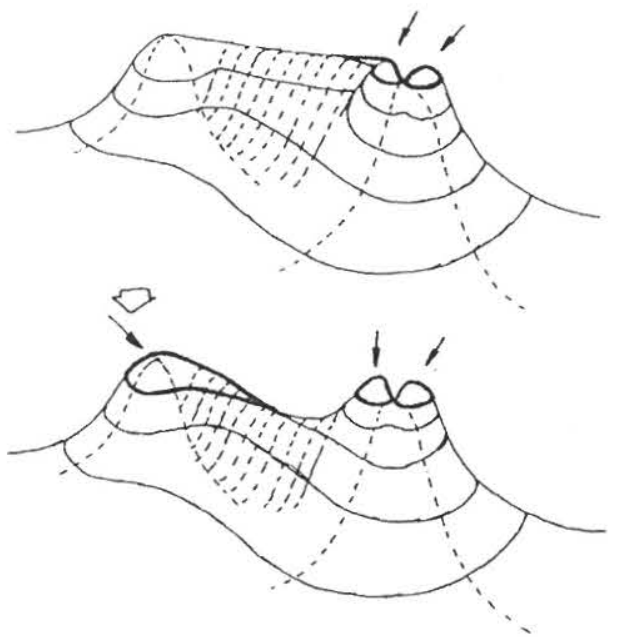

Figure 4: (a) Example illustrating the property that new local extrema can be created with increasing scale in the scale-space representation of a two-dimensional signal. Interpreted in terms of blobs the configuration describes a blob split. (b) By modifying the example slightly (replacing the higher one of the two peaks with a double peak) one realizes that blob creations can occur as well.

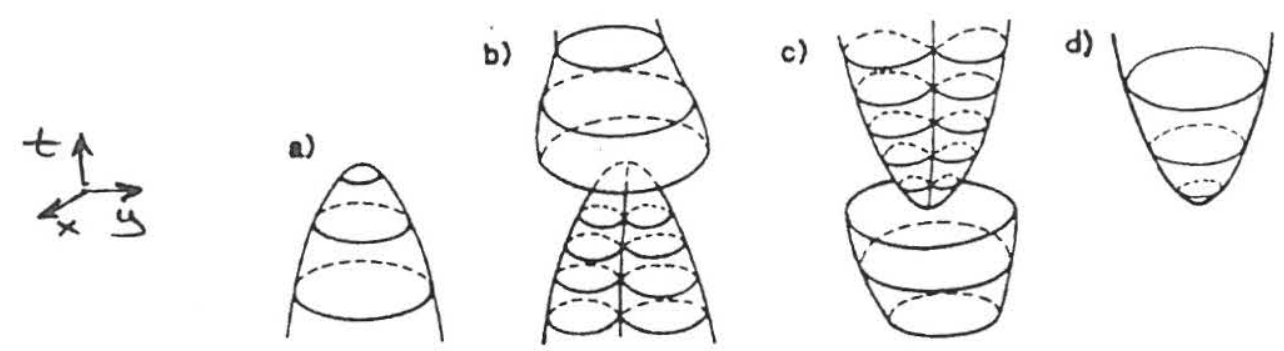

Figure 5: Illustration of the various events possible for the scale-space blobs of a two-dimensional signal: (a) blob annihilation (b) blob merge (c) blob split (d) blob creation. Note that the grey-level coordinate of the blobs has been omitted. Hence, only the support regions of the grey-level blobs are displayed.

at the same moment. Such events will, however, be unstable since even the slightest perturbation of the input signal would perturb such a simultaneous merge of three blobs into a sequence of two successive pairwise merges. Note in this context that for Morse functions, see e.g. Arnold [1], no pair of critical points will have the same values. In other words, for generic functions all critical points will be distinct. Although, by definition, the grey-level function will not be Morse at a bifurcation, we can, in general, assume this latter property to hold at bifurcations, which means that situations with three or more blobs simultaneously merging into one, can in general be expected not to occur.

For one-dimensional signals the analogies of Definition 2 and Proposition 10 will be as follows: We express the formulations for bright blobs only. The case with dark blobs is similar.

Definition 3 (Non-shared extremum path (bright blobs in 1D))

Consider the case with bright blobs in the scale-space representation of a one-dimensional signal. Given an extremum path of minimum points involved in a structurally stable bifurcation we say that the extremum path is non-shared before (after) the bifurcation if there exists some scale interval before (after) the bifurcation during which every minimum point of the extremum path is contained in only one bright grey-level blob. Otherwise the saddle path is said to be shared.

Proposition 11 (Scale-space blob events (bright blobs in 1D))

In the scale-space representation of a one-dimensional continuous signal, the following events between bright blobs are possible at a structurally stable bifurcation:

- blob annihilation - annihilation of a minimum-maximum pair where the extremum path of minimum points is non-shared before the bifurcation.

- blob merge - annihilation of an extremum-saddle pair where the extremum path of minimum points is shared with another bright scale-space blob before the bifurcation. 
Proof: From Corollary 9 we have that the typical behaviour at singularities in a one-parameter family of functions are pairwise annihilations and creations of minimum-maximum pairs. Since, however, the number of local extrema cannot increase with scale in the scale-space representation of a one-dimensional signal it follows that new minimum-maximum pairs cannot arise with increasing scale. This means that blob splits and blob creations are impossible in one dimension because of the causality requirements. $\diamond$

These bifurcations define the hierarchical relations across scales between scale-space blobs at different scales in the tree-like representation called scale-space primal sketch, $[11,12]$. The data structure generated from these objects will, however, not constitute a tree in a strict sense because more than one annihilation and also splits can occur in scale-space.

\section{Summary}

We have analysed the behaviour of critical points in scale-space and shown that non-degenerate critical points will in general form regular curves across scales. Along those we have provided generally valid estimates of the drift velocity. At degenerate critical points the behaviour is more complicated and bifurcations may take place. For one-dimensional signals, the only bifurcation events possible when the scale parameter increases, are annihilations of pairs of local maxima and minima, while for two-dimensional signals both annihilations and creations of pairs of local extrema and saddle points can occur. Applied to grey-level and scale-space blobs only annihilations and merges will take place in the one-dimensional case, while the list of possibilities in two-dimensions comprises four types: annihilations, merges, splits and creations.

\section{References}

[1] Arnold V.I. (1981) Singularity Theory, Selected papers, London Math. Soc. Lect. Note Ser., 53, Cambridge Univ. Press, Cambridge.

[2] Arnold V.I., Gusein-Zade S.M., Varchenko A.N. $(1985,1988)$ Singularities of Smooth Maps, Volume $I, I I$, Birkhäuser, Boston.

[3] Babaud J., Witkin A.P., Baudin M. and Duda R.O. (1986) "Uniqueness of the Gaussian Kernel for Scale-Space Filtering", IEEE-PAMI, 8:1, 26-33.

[4] Bergholm F. (1987) "Edge Focusing", IEEE-PAMI, 9:6, 726-741.

[5] Berzins V. (1984) "Accuracy of Laplacian Edge Detectors", CVGIP, 27, 195-210.

[6] Canny J. (1986) "A Computational Approach to Edge Detection", IEEE-PAMI, 8:6, .

[7] Koenderink J.J., van Doorn A.J. (1984) "The Structure of Images", Biol. Cyb., 50, 363-370.

[8] Koenderink J.J., van Doorn A.J. (1986) "Dynamic Shape", Biol. Cyb., 53, pp383-396.

[9] Lifshitz L.M., Pizer S.M. (1987) "A Multiresolution Hierarchical Approach to Image Segmentation Based on Intensity Extrema", Internal report, Depts. of Comp. Sci. and Radiology, Univ. of North Carolina, Chapel Hill, N.C., U.S.A.

[10] Lindeberg T.P. (1990) "Scale-Space for Discrete Signals", IEEE-PAMI, 12:3, 234-254.

[11] Lindeberg T.P., Eklundh J.O. (1990) "On the Computation of a Scale-Space Primal Sketch", To appear in Journal of Visual Communication and Image Representation. A short version also in Proc. British Machine Vision Conference 1990, Oxford, Great Britain.

[12] Lindeberg T.P., Eklundh J.O. (1990) "Scale Detection and Region Extraction from a Scale-Space Primal Sketch", To appear in 3:rd ICCV, Osaka, Japan.

[13] Lindeberg T.P., Eklundh J.O. (1990) "Guiding Early Visual Processing with a Scale-Space Primal Sketch", Submitted.

[14] Lindeberg T.P. (1991) In preparation.

[15] Poston T., Stewart I. (1978) "Catastrophe Theory and its Applications", Pitman Publishing, London.

[16] Witkin A.P. (1983) "Scale-Space Filtering", Proc. 7:th IJCAI, Karlsruhe, 1019-1022.

[17] Zhang W., Bergholm F. (1990) "An Extension to Marr's Signature Based Edge Classification", CVAP-TN-04, Dept. of Num. Anal. and Comp. Sci., Royal Institute of Technology, Stockholm. 